\title{
DISCOVERY OF HIGHLY OBSCURED GALAXIES IN THE ZONE OF AVOIDANCE
}

\author{
F. R. Marleau ${ }^{1}$, A. Noriega-Crespo ${ }^{1}$, R. Paladini ${ }^{1}$, D. Clancy $^{1}$, S. Carey ${ }^{1}$, S. Shenoy ${ }^{1}$, K. E. Kraemer ${ }^{2}$, T. Kuchar ${ }^{3}$, \\ D. R. Mizuno ${ }^{3}$, ANd S. PRICE ${ }^{2}$ \\ ${ }^{1}$ Spitzer Science Center, California Institute of Technology, CA 91125, USA \\ ${ }^{2}$ Air Force Research Laboratory, Hanscom AFB, MA 01731, USA \\ ${ }^{3}$ Institute for Scientific Research, Boston College, Boston, MA 02135, USA \\ Received 2007 September 25; accepted 2008 May 6; published 2008 July 8
}

\begin{abstract}
We report the discovery of 25 previously unknown galaxies in the zone of avoidance (ZoA). Our systematic search for extended extragalactic sources in the Galactic Legacy Infrared Mid-Plane Survey Extraordinaire (GLIMPSE) and MIPS Galactic Plane Survey (MIPSGAL) mid-infrared surveys of the Galactic plane has revealed two overdensities of these sources, located around $l \sim 47^{\circ}$ and $55^{\circ}$ and $|b| \lesssim 1^{\circ}$ in the Sagitta-Aquila region. These overdensities are consistent with the local large-scale structure found at similar Galactic longitude and extending from $|b| \sim 4$ to $40^{\circ}$. We show that the infrared spectral energy distribution of these sources is indeed consistent with those of normal galaxies. Photometric estimates of their redshift indicate that the majority of these galaxies are found in the redshift range $z \simeq 0.01-0.05$, with one source located at $z \simeq 0.07$. Comparison with known sources in the local universe reveals that these galaxies are located at similar overdensities in redshift space. These new galaxies are the first evidence of a bridge linking the large-scale structure between both sides of the Galactic plane at very low Galactic latitude and clearly demonstrate the feasibility of detecting galaxies in the ZoA using mid-to-far infrared surveys.
\end{abstract}

Key words: galaxies: distances and redshifts - infrared: galaxies - large-scale structure of universe

\section{INTRODUCTION}

The last frontier in mapping the large-scale structure of the local universe is the zone of avoidance (ZoA). Starting in the late 1980s, redshift surveys have provided an increasingly detailed picture of the large-scale structure in the northern and southern Galactic hemispheres, above and below the ZoA (e.g., CfA Redshift Survey, Geller \& Huchra 1989; 2dF Redshift Survey, Colless 1999; Las Campanas Redshift Survey, Shectman et al. 1996). However, the precise way the coherent structures of the two halves connect remains largely unknown and is not trivially predictable from the existing data. Moreover, it is quite likely that undiscovered structures incorporating significant mass concentrations lie behind the ZoA, as appears to be the case in the region of the "Great Attractor" (Galactic longitude $l \sim 320^{\circ}$ and latitude $b \sim 0^{\circ}$; Kolatt et al. 1995), and finding these is essential for determining the dynamics of our local universe.

Many dedicated surveys of the ZoA have searched for hidden mass concentrations of galaxies (see reviews by Kraan-Korteweg \& Lahav 2000; Kraan-Korteweg 2005). These have been primarily undertaken in the optical (e.g., Roman et al. 2000), near-infrared (DENIS, Schröder et al. 1999; Two Micron All Sky Survey (2MASS), Jarrett et al. 2000), farinfrared (IRAS; Takata et al. 1996), and HI/radio (Parkes; see e.g. Henning et al. 2000), resulting in a considerable reduction of the ZoA. For example, the southern hemisphere HI survey with Parkes was able to map over a thousand new galaxies within $|b|<5^{\circ}$, extending the prominence of the Norma supercluster (Radburn-Smith et al. 2006). The most heavily investigated region, using the widest wavelength regimes, has been the Great Attractor region, where a large mass overdensity of $5 \times 10^{16}$ solar masses has been predicted from the systematic infall of 400 ellipticals (Dressler et al. 1987). However, the most obscured regions $\left(|b| \leqslant 1^{\circ}\right)$ of the Milky Way, with visual extinction larger than 13 mag, remain largely unexplored.
Recently, the Spitzer Space Telescope opened the door to further advances and discoveries in the ZoA, with its significantly improved detector technology, providing both better resolution and sensitivity at mid- to far-infrared wavelengths (Werner et al. 2004). Indeed, one of the first large surveys conducted by Spitzer was the Galactic Legacy Infrared Mid-Plane Survey Extraordinaire (GLIMPSE; Benjamin et al. 2003). GLIMPSE surveyed $2 / 3$ of the inner Galactic disk with a pixel resolution of $1.2^{\prime \prime}$, using the Infrared Array Camera (IRAC) at 3.6, 4.5, 5.8, and $8.0 \mu \mathrm{m}$. The survey covered Galactic latitudes $|b|<1^{\circ}$ and longitudes $|l|=10-65^{\circ}$, both sides of the Galactic center. Source confusion and saturation from the bright star-forming regions forced the GLIMPSE team to adopt a relatively conservative approach, mapping the plane with a $4 \mathrm{~s}$ integration time. In comparison, the Galactic First Look Survey (FLS; Burgdorf et al. 2005) used $48 \mathrm{~s}$ exposures at $l=97^{\circ}$ and $|b|<4^{\circ}$, to study the structure of the Galactic disk. The following year, the GLIMPSE survey was complemented by the 24 and 70 Micron Survey of the Inner Galactic Disk (MIPSGAL; Carey et al. 2008, in preparation), essentially covering the same region of the plane at longer wavelengths. One of the first extragalactic results that followed from these dust-penetrating surveys was the discovery of galaxies peering through the most obscured portion of the Great Attractor (Jarrett et al. 2007), demonstrating the discovery potential of Spitzer in the ZoA.

\section{DATA}

In this paper, we present the results of a galaxy search in the ZoA based on data obtained from the two Spitzer Legacy Surveys of the Galactic plane: the GLIMPSE (Benjamin et al. 2003) and the MIPS Galactic Plane Survey (MIPSGAL) (Carey et al. 2008). These surveys cover a similar region, i.e., Galactic latitudes $|l|=10^{\circ}-65^{\circ}$ and one degree longitude above and below the plane $\left(|b| \lesssim 1^{\circ}\right)$.

The enhanced products from GLIMPSE were downloaded directly from the Spitzer Science Center popular products Web 
Table 1

Coordinates of Spitzer Galaxies in the ZoA

\begin{tabular}{|c|c|c|c|c|c|}
\hline ID & Name & $\alpha_{J 2000}$ & $\delta_{J 2000}$ & $l$ & $b$ \\
\hline 1 & SPITZER191050+113409a & $19^{\mathrm{h}} 10^{\mathrm{m}} 50.432$ & $11^{\circ} 34^{\prime} 09^{\prime \prime} .38$ & 45.42353 & 1.01550 \\
\hline 2 & SPITZER192252+124513 & $19^{\mathrm{h}} 22^{\mathrm{m}} 52^{\mathrm{s}} .169$ & $12^{\circ} 45^{\prime} 12^{\prime \prime} .84$ & 47.84276 & -1.03199 \\
\hline 3 & SPITZER192404+145632 & $19^{\mathrm{h}} 24^{\mathrm{m}} 04^{\mathrm{s}} .473$ & $14^{\circ} 56^{\prime} 31^{\prime \prime} .75$ & 49.91004 & -0.25584 \\
\hline 4 & SPITZER193206+183140 & $19^{\mathrm{h}} 32^{\mathrm{m}} 06^{\mathrm{s}} .060$ & $18^{\circ} 31^{\prime} 39^{\prime \prime} .88$ & 53.97787 & -0.23159 \\
\hline 5 & SPITZER193145+181940 & $19^{\mathrm{h}} 31^{\mathrm{m}} 44^{\mathrm{s}} .840$ & $18^{\circ} 19^{\prime} 39^{\prime \prime} .88$ & 53.76221 & -0.25451 \\
\hline 6 & SPITZER193133+181516 & $19^{\mathrm{h}} 31^{\mathrm{m}} 32^{\mathrm{s}} 750$ & $18^{\circ} 15^{\prime} 16^{\prime \prime} .45$ & 53.67504 & -0.24784 \\
\hline 7 & SPITZER193105+182614 & $19^{\mathrm{h}} 31^{\mathrm{m}} 04^{\mathrm{s}} .748$ & $18^{\circ} 26^{\prime} 14^{\prime \prime} .12$ & 53.78187 & -0.06284 \\
\hline 8 & SPITZER193337+190727 c & $19^{\mathrm{h}} 33^{\mathrm{m}} 36^{\mathrm{s}} .564$ & $19^{\circ} 07^{\prime} 27^{\prime \prime} .23$ & 54.67258 & -0.25585 \\
\hline 9 & SPITZER193320+193334 & $19^{\mathrm{h}} 33^{\mathrm{m}} 20^{\mathrm{s}} 301$ & $19^{\circ} 33^{\prime} 34^{\prime \prime} .38$ & 55.02256 & 0.01080 \\
\hline 10 & SPITZER193349+202958 ${ }^{\mathrm{d}}$ & $19^{\mathrm{h}} 33^{\mathrm{m}} 48.593$ & $20^{\circ} 29^{\prime} 58^{\prime \prime} .49$ & 55.89871 & 0.36900 \\
\hline 11 & SPITZER193915+201426 & $19^{\mathrm{h}} 39^{\mathrm{m}} 14^{\mathrm{s}} .703$ & $20^{\circ} 14^{\prime} 25^{\prime \prime} .88$ & 56.29427 & -0.86925 \\
\hline 12 & SPITZER193825+212701 & $19^{\mathrm{h}} 38^{\mathrm{m}} 24^{\mathrm{s}} .972$ & $21^{\circ} 27^{\prime} 01^{\prime \prime} .29$ & 57.25305 & -0.10634 \\
\hline 13 & SPITZER193324+214918 & $19^{\mathrm{h}} 33^{\mathrm{m}} 24^{\mathrm{s}} .468$ & $21^{\circ} 49^{\prime} 17^{\prime \prime} .93$ & 57.01030 & 1.09097 \\
\hline 14 & SPITZER193956+213017 & $19^{\mathrm{h}} 39^{\mathrm{m}} 56^{\mathrm{s}} .106$ & $21^{\circ} 30^{\prime} 17^{\prime \prime} .31$ & 57.47421 & -0.38725 \\
\hline 15 & SPITZER194223+212514 & $19^{\mathrm{h}} 42^{\mathrm{m}} 23.331$ & $21^{\circ} 25^{\prime} 13^{\prime \prime} .72$ & 57.68324 & -0.92517 \\
\hline 16 & SPITZER193633+225125 & $19^{\mathrm{h}} 36^{\mathrm{m}} 32^{\mathrm{s}} .781$ & $22^{\circ} 51^{\prime} 25^{\prime \prime} .18$ & 58.26802 & 0.95949 \\
\hline 17 & SPITZER193608+225054 ${ }^{\mathrm{f}}$ & $19^{\mathrm{h}} 36^{\mathrm{m}} 07^{\mathrm{s}} .572$ & $22^{\circ} 50^{\prime} 54^{\prime \prime} .33$ & 58.21334 & 1.03982 \\
\hline 18 & SPITZER193632+225355 & $19^{\mathrm{h}} 36^{\mathrm{m}} 31^{\mathrm{s}} .917$ & $22^{\circ} 53^{\prime} 54^{\prime \prime} .81$ & 58.30269 & 0.98266 \\
\hline 19 & SPITZER193958+235251 ${ }^{\mathrm{g}}$ & $19^{\mathrm{h}} 39^{\mathrm{m}} 58^{\mathrm{s}} .334$ & $23^{\circ} 52^{\prime} 50^{\prime \prime} .69$ & 59.54589 & 0.77617 \\
\hline 20 & SPITZER194042+232429 & $19^{\mathrm{h}} 40^{\mathrm{m}} 42^{\mathrm{s}} .470$ & $23^{\circ} 24^{\prime} 29^{\prime \prime} .33$ & 59.21788 & 0.39649 \\
\hline 21 & SPITZER194211+242507 & $19^{\mathrm{h}} 42^{\mathrm{m}} 10.975$ & $24^{\circ} 25^{\prime} 06^{\prime \prime} .66$ & 60.26274 & 0.60358 \\
\hline 22 & SPITZER194257+251849 & $19^{\mathrm{h}} 42^{\mathrm{m}} 57^{\mathrm{s}} .449$ & $25^{\circ} 18^{\prime} 49^{\prime \prime} .18$ & 61.12754 & 0.89557 \\
\hline 23 & SPITZER194349+251449 & $19^{\mathrm{h}} 43^{\mathrm{m}} 48^{\mathrm{s}} .966$ & $25^{\circ} 14^{\prime} 49^{\prime \prime} .40$ & 61.16589 & 0.69407 \\
\hline 24 & SPITZER194919+243738 & $19^{\mathrm{h}} 49^{\mathrm{m}} 19.339$ & $24^{\circ} 37^{\prime} 37^{\prime \prime} .64$ & 61.25773 & -0.69658 \\
\hline 25 & SPITZER195443+260745 & $19^{\mathrm{h}} 54^{\mathrm{m}} 43^{\mathrm{s}} .154$ & $26^{\circ} 07^{\prime} 45^{\prime \prime} .08$ & 63.17221 & -0.97524 \\
\hline
\end{tabular}

Notes.

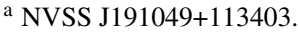

b 1WGA J1931.7+1818.

c NVSS J193336+190723.

${ }^{d}$ NVSS J193349+203131.

e WSRTGP $1937+2122$.

f WSRTGP 1933+2243.

g NVSS J193958+235243.

site, ${ }^{4}$ and included post-processed mosaics of the four IRAC (Fazio et al. 2004) bands (3.6, 4.5, 5.8, and $8 \mu \mathrm{m}$ ), as well as a detailed description on how the data were reduced (Meade et al. 2007a, 2007b). The GLIMPSE data were taken using two $2 \mathrm{~s}$ exposures in order to deal with some of the brightest regions in the Galactic plane, and therefore the images remained shallow in the darkest regions. The quoted surface brightness sensitivities $(5 \sigma)$ were $2.7,2.2,6.3$, and $5.6 \mathrm{mJy} \mathrm{sr}^{-1}$ at 3.6 , $4.5,6.0$, and $8 \mu \mathrm{m}$, respectively. The angular resolution of the enhanced mosaics was $\sim 2.5^{\prime \prime}$, with a plate size of one square degree.

The MIPSGAL data were obtained using MIPS (Rieke et al. 2004) in its fast scan mode. The mapping at $24 \mu \mathrm{m}$ relied on two passes, for a minimum coverage of ten samples per position, with a total integration time of $30 \mathrm{~s}$, and with an extended source sensitivity of $\sim 0.16 \mathrm{MJy} \mathrm{sr}^{-1}(1 \sigma)$. This scanning strategy was chosen so as to compensate for the latent effects produced by bright sources. Due to the smaller field of view (FOV) of the $70 \mu \mathrm{m}$ array compared to that at $24 \mu \mathrm{m}\left(\sim 2^{\prime} \cdot 5 \times 5^{\prime}\right.$ versus $\left.5^{\prime} \times 5^{\prime}\right)$, the data were obtained using an interlaced pattern with a minimum coverage of five samples per pixel, yielding a total integration time of $15 \mathrm{~s}$ and a sensitivity of $\sim 0.74 \mathrm{MJy} \mathrm{sr} r^{-1}$ $(1 \sigma)$. The enhanced products, that will soon be delivered and released (Carey et al. 2008), included post-processed mosaics at 24 and $70 \mu \mathrm{m}$, with an angular resolution of $\sim 6^{\prime \prime}$ and $20^{\prime \prime}$, respectively, and a plate size of 1.21 square degree $\left(1.1^{\circ} \times 1.1^{\circ}\right)$.
The IRAC and MIPS data were supplemented with nearinfrared $J, H$ and $K \mathrm{~s}(1.25,1.65$, and $2.17 \mu \mathrm{m}$, respectively) data from the 2MASS (Skrutskie et al. 2006), downloaded directly from the NASA/IPAC Infrared Science Archive (IRSA). ${ }^{5}$

The boundaries of our search were set by the available processed MIPSGAL data and the structure of the Galactic plane itself (avoiding very bright and confused regions), leaving us with approximately 50 square degrees $\left(40^{\circ} \geqslant l \geqslant 65^{\circ} ;-1 \geqslant\right.$ $b \geqslant 1$ ) to survey during our initial search. Our visual search for extended sources in the $24 \mu \mathrm{m}$ MIPSGAL data revealed the first galaxy candidate. In continuing our search, we found that using the GLIMPSE enhanced product, with higher angular resolution, was a better way to identify potential galaxy candidates. We made use of the red color (in the composite IRAC 3.6, 4.5, and $8 \mu \mathrm{m}$ images) of these galaxies to narrow down our search in the GLIMPSE plates.

\section{DETECTION OF GALAXIES AND CROSS-IDENTIFICATION}

Our systematic search, covering $\sim 25^{\circ}$ in Galactic longitude, successfully revealed two overdensities of a total of 25 new galaxies, located around $l \sim 47^{\circ}$ and $55^{\circ}$ in the Sagitta-Aquila region (see Table 1 and Figure 1). Their IRAC 3.5, 4.5, and $8 \mu \mathrm{m}$ color composite images are shown in Figure 2. Of these, 22 were found to have a $24 \mu \mathrm{m}$ counterpart in the MIPSGAL images,

\footnotetext{
4 http://data.spitzer.caltech.edu/popular/glimpse/.
}

5 http://irsa.ipac.caltech.edu/Missions/2mass.html. 

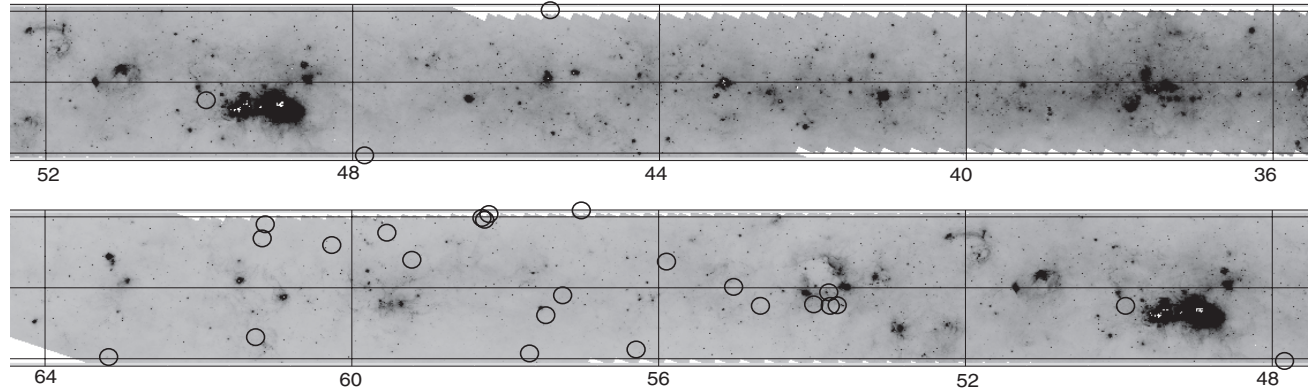

Figure 1. Location of the twenty-five newly discovered galaxies, superposed on the $24 \mu \mathrm{m}$ MIPSGAL image ( $5^{\prime \prime}$ per pixel) of the Galactic plane. The top panel covers $l \sim 36^{\circ}-52^{\circ}$ and the bottom one $l \sim 48^{\circ}-64^{\circ}$, with $|b| \lesssim 1^{\circ}$.
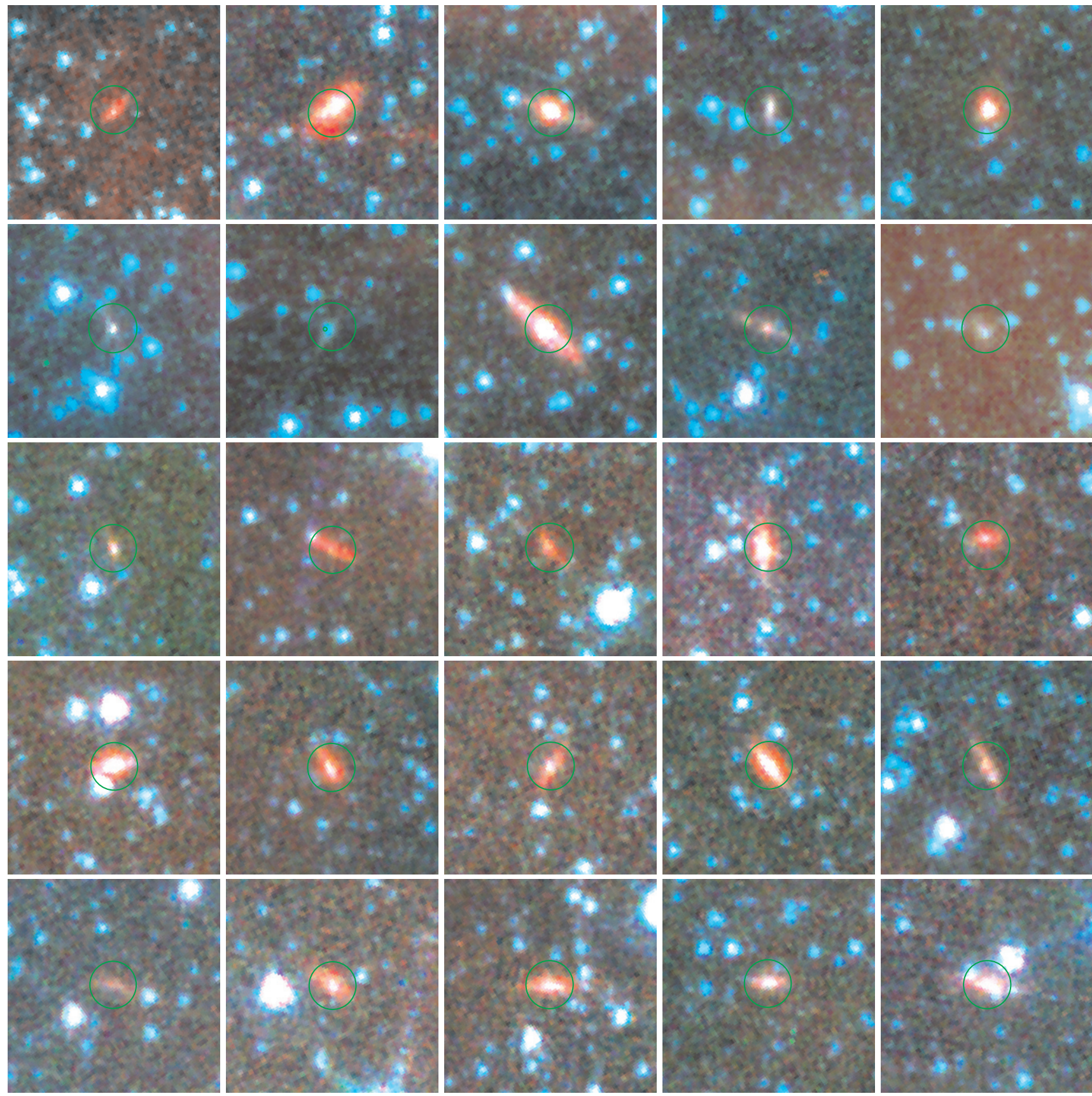

Figure 2. IRAC 3.5 (blue), 4.5 (green), and $8 \mu \mathrm{m}$ (red) color composite postage stamps of our sample of 25 newly discovered galaxies. The galaxies are ordered from top to bottom and left to right following the listing order in Table 1. Most of these galaxies are at the edge of the GLIMPSE survey detection limit. The FOV of each image is $53^{\prime \prime} \times 53^{\prime \prime}\left(0.6^{\prime \prime}\right.$ per pixel) with north up and east to the left. The green circles overlaid on the images are $12^{\prime \prime}$ diameter in size. The scale parameters of the postage stamps were optimized for each individual source and therefore differ for each image. 

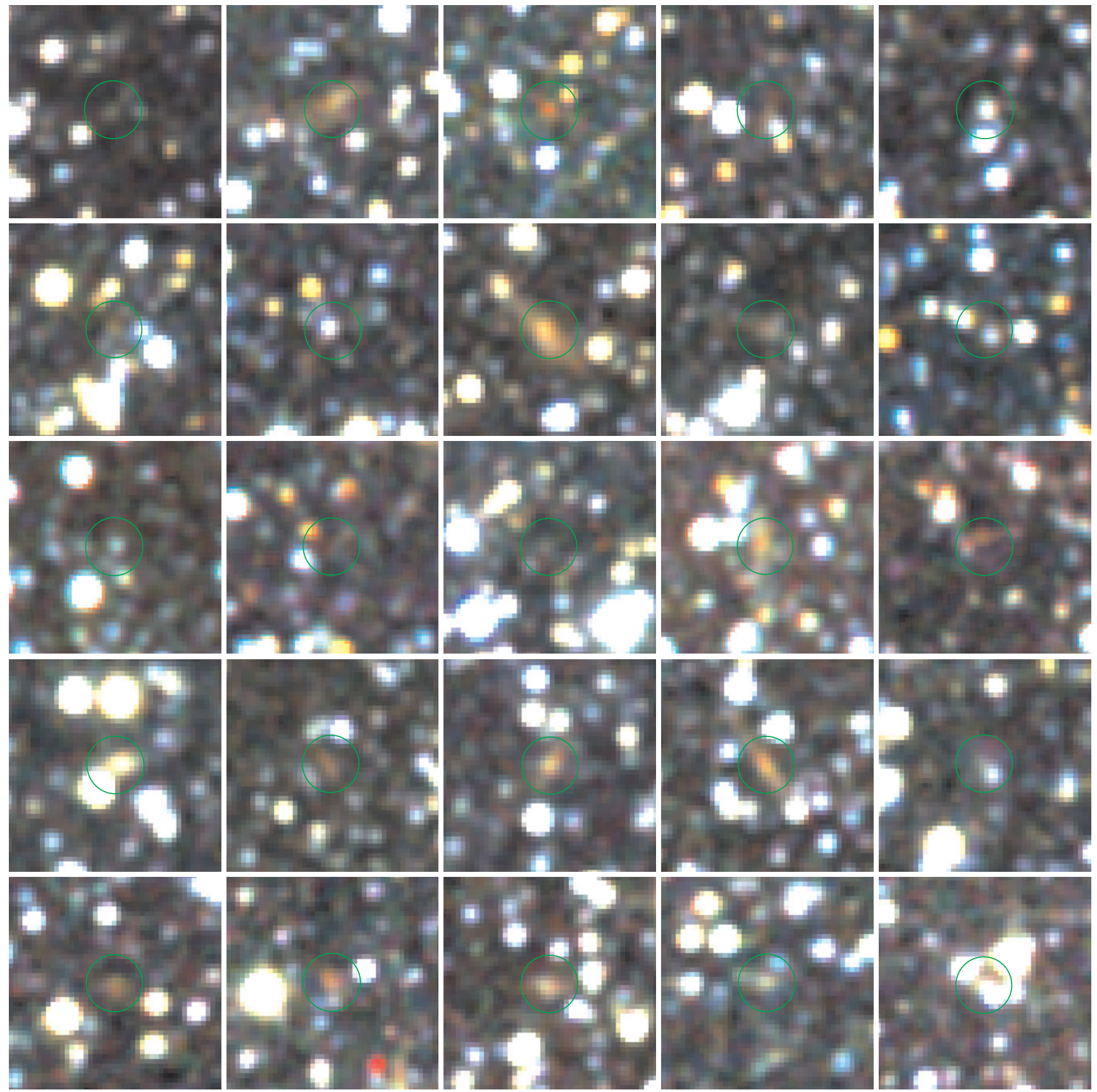

Figure 3. 2MASS $J$ (blue), $H$ (green), and $K$ s (red) color composite images of the same galaxies. The galaxies are displayed in the same order as shown in Figure 2 . These extended sources are barely visible in the near-infrared where the extinction is higher, as seen in these images. The FOV of each image is $43^{\prime \prime} \times 43^{\prime \prime}\left(0.99972^{\prime \prime}\right.$ per pixel) with north up and east to the left. The green circles overlaid on the images are $12^{\prime \prime}$ diameter in size. The scale parameters of the postage stamps were optimized for each individual source and therefore differ for each image.

as three fell outside the MIPS survey and nine had measurable flux densities at $70 \mu \mathrm{m}$.

We cross-identified our candidate galaxies with their 2MASS $J, H$, and $K$ s counterparts (see Figure 3 ). As can be seen in Figures 2 and 3, our galaxies are typically very faint in all IRAC bands (median value of IRAC $8 \mu \mathrm{m}=8.9 \mathrm{mag}$ ), and just barely visible in the 2MASS near-infrared images (median value of $K$ s $2.17 \mu \mathrm{m}=11.9 \mathrm{mag}$ ).

\section{PHOTOMETRIC MEASUREMENTS}

The spectral energy distribution (SED) of each candidate galaxy was derived using photometric measurements obtained in all nine bands (from 1.25 to $70 \mu \mathrm{m}$ ). The aperture position and size for each object were fixed in order to measure the colors or broadband SED at the same physical region of each galaxy. The photometry was done using an aperture radius of $6^{\prime \prime}$ and the background contributing to the total flux density within the same aperture was removed. This aperture size was chosen to avoid as much as possible contamination due to foreground stars, mostly seen in the near-infrared images, while matching the average size, i.e. two times the full width at half-maximum (FWHM), of the point-spread function (PSF) at $24 \mu \mathrm{m}$ (FWHM of $6^{\prime \prime}$ ) given that all candidate galaxies are essentially point sources at $24 \mu \mathrm{m}$ (see Figure 4). Nevertheless, the flux density measurements of some galaxies, e.g. SPITZER193633+225125, had to be corrected due to contamination from foreground stars. 


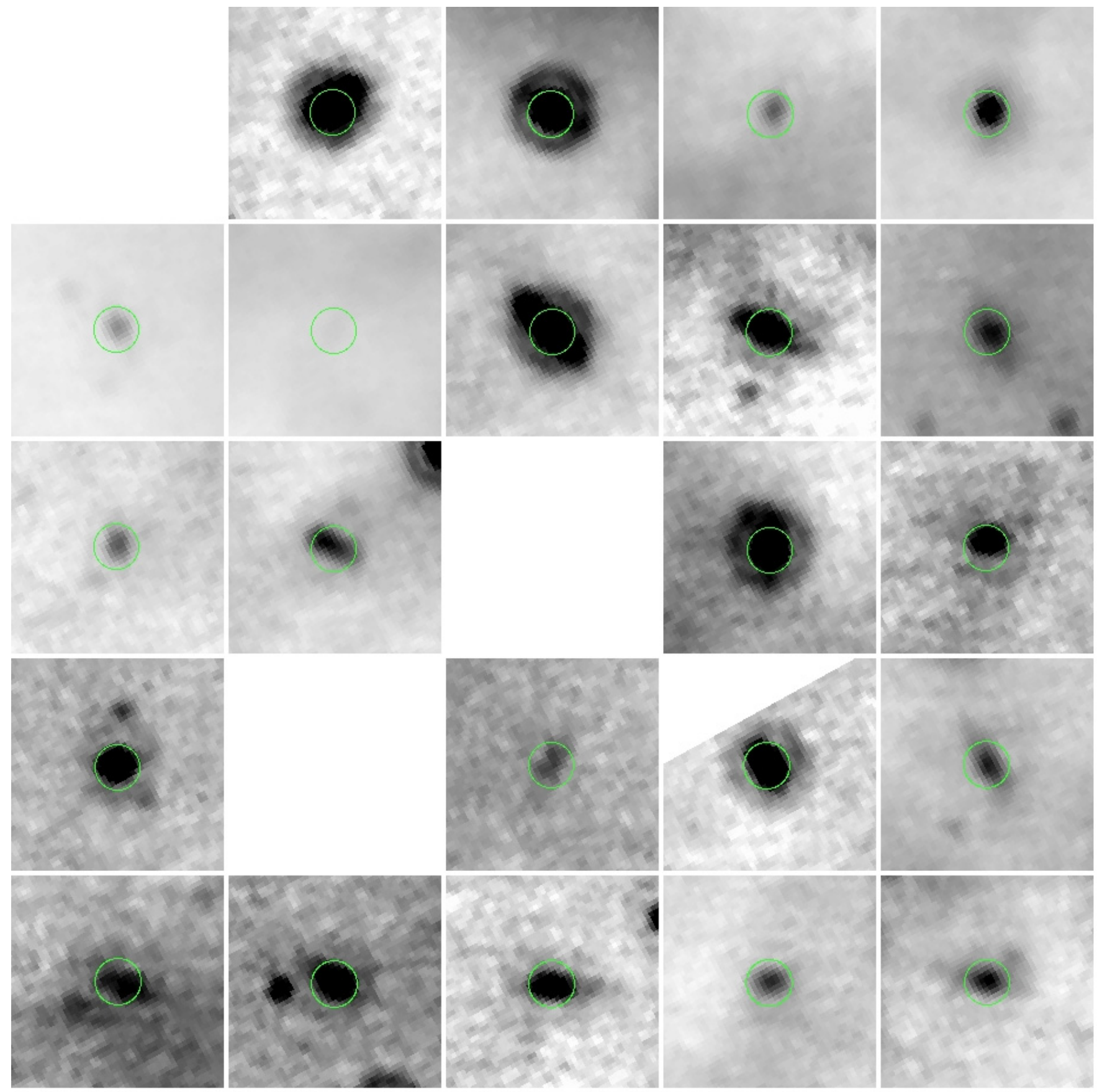

Figure 4. MIPS $24 \mu \mathrm{m}$ postage stamps of the same galaxies. The galaxies are displayed in the same order as shown in Figure 2. The candidate galaxies are essentially point sources at $24 \mu \mathrm{m}$. The FOV of each image is $54^{\prime \prime} \times 54^{\prime \prime}\left(1.25^{\prime \prime}\right.$ per pixel $)$ with north up and east to the left. The green circles overlaid on the images are $12^{\prime \prime}$ diameter in size. The images are displayed in reverse color map and the scale parameters of the postage stamps were optimized for each individual source and therefore differ for each image.

This was done by interpolating over the neighboring galaxy pixel values.

For the 2MASS measurements, a zero point was estimated using a point source in the field with photometric measurements provided by the 2MASS point source catalog. ${ }^{6}$ No aperture correction was applied as it is essentially negligible. ${ }^{7}$ Dust extinction toward each galaxy was inferred from the IRAS/ DIRBE far-infrared maps (Schlegel et al. 1998) (see Table 2). The values of $A(\mathrm{~V})$ at the reference point were transformed

\footnotetext{
6 http://irsa.ipac.caltech.edu/.

7 see http://www.ipac.caltech.edu/2mass/releases/allsky/doc/sec4_4c.html.
}

to the 2MASS passbands using the extinction curve of Dutra et al. (2002), where $A(\mathrm{~J})=0.301 A(\mathrm{~V}), A(\mathrm{H})=0.180 A(\mathrm{~V})$, and $A(\mathrm{Ks})=0.118 A(\mathrm{~V})$. The values of $A(\mathrm{Ks})$ for the candidate galaxies range from 0.9 to $3.7 \mathrm{mag}$.

The IRAC flux densities were multiplied by the aperture correction factor according to the formulation derived by the IRAC team. ${ }^{8}$ The aperture correction for an extended source and for a $6 "$ radius aperture was 1.02778 (channel 1), 1.07868 (channel 2), 1.00985 (channel 3), and 0.965037 (channel 4). The zero magnitude flux densities were taken from Reach

\footnotetext{
8 http://ssc.spitzer.caltech.edu/irac/calib/extcal/.
} 
Table 2

Extinction Correction for Photometric Measurements of Spitzer Galaxies in the ZoA

\begin{tabular}{rrrrrcccccccc}
\hline \hline ID & $A(\mathrm{~V})^{\mathrm{a}}$ & $A(\mathrm{~J})$ & $A(\mathrm{H})$ & $A(\mathrm{Ks})$ & $A(\mathrm{I} 1)$ & $A(\mathrm{I} 2)$ & $A(\mathrm{I} 3)$ & $A(\mathrm{I} 4)$ & $A(\mathrm{M} 1)$ & $A(\mathrm{M} 2)$ & $A(\mathrm{~V})$ (SED fit $)$ \\
& $0.5 \mu \mathrm{m}$ & $1.25 \mu \mathrm{m}$ & $1.65 \mu \mathrm{m}$ & $2.17 \mu \mathrm{m}$ & $3.6 \mu \mathrm{m}$ & $4.5 \mu \mathrm{m}$ & $5.8 \mu \mathrm{m}$ & $8.0 \mu \mathrm{m}$ & $24.0 \mu \mathrm{m}$ & $70.0 \mu \mathrm{m}$ & $0.5 \mu \mathrm{m}$ \\
\hline 1 & 8.9 & 2.7 & 1.6 & 1.0 & 0.6 & 0.5 & 0.4 & 0.4 & 0.1 & 0.02 & 29.8 \\
2 & 14.2 & 4.3 & 2.6 & 1.7 & 0.9 & 0.8 & 0.7 & 0.7 & 0.2 & 0.03 & 17.3 \\
3 & 22.6 & 6.8 & 4.1 & 2.7 & 1.5 & 1.3 & 1.1 & 1.1 & 0.3 & 0.04 & 26.1 \\
4 & 23.0 & 6.9 & 4.1 & 2.7 & 1.5 & 1.3 & 1.1 & 1.1 & 0.3 & 0.04 & 20.2 \\
5 & 17.1 & 5.2 & 3.1 & 2.0 & 1.1 & 1.0 & 0.8 & 0.8 & 0.2 & 0.03 & 16.9 \\
6 & 19.5 & 5.9 & 3.5 & 2.3 & 1.3 & 1.1 & 1.0 & 0.9 & 0.3 & 0.04 & 17.8 \\
7 & 31.0 & 9.3 & 5.6 & 3.7 & 2.0 & 1.7 & 1.5 & 1.5 & 0.4 & 0.06 & 16.3 \\
8 & 13.8 & 4.2 & 2.5 & 1.6 & 0.9 & 0.7 & 0.7 & 0.7 & 0.2 & 0.02 & 15.9 \\
9 & 17.6 & 5.3 & 3.2 & 2.1 & 1.2 & 1.0 & 0.8 & 0.8 & 0.2 & 0.03 & 21.6 \\
10 & 23.8 & 7.2 & 4.3 & 2.8 & 1.6 & 1.3 & 1.2 & 1.1 & 0.3 & 0.04 & 17.1 \\
11 & 11.6 & 3.5 & 2.1 & 1.4 & 0.8 & 0.7 & 0.6 & 0.5 & 0.2 & 0.02 & 22.2 \\
12 & 28.2 & 8.5 & 5.1 & 3.3 & 1.9 & 1.6 & 1.4 & 1.3 & 0.4 & 0.05 & 28.3 \\
13 & 14.5 & 4.3 & 2.6 & 1.7 & 1.0 & 0.8 & 0.7 & 0.7 & 0.2 & 0.03 & 26.0 \\
14 & 21.7 & 6.6 & 3.9 & 2.6 & 1.4 & 1.2 & 1.1 & 1.0 & 0.3 & 0.04 & 23.7 \\
15 & 11.9 & 3.6 & 2.2 & 1.4 & 0.8 & 0.7 & 0.6 & 0.5 & 0.2 & 0.02 & 17.0 \\
16 & 11.6 & 3.5 & 2.1 & 1.4 & 0.8 & 0.6 & 0.6 & 0.5 & 0.2 & 0.02 & 5.5 \\
17 & 10.8 & 3.2 & 1.9 & 1.3 & 0.7 & 0.6 & 0.5 & 0.5 & 0.1 & 0.02 & 12.6 \\
18 & 10.7 & 3.2 & 1.9 & 1.3 & 0.7 & 0.6 & 0.5 & 0.5 & 0.1 & 0.02 & 9.3 \\
19 & 9.6 & 2.9 & 1.7 & 1.1 & 0.6 & 0.5 & 0.5 & 0.4 & 0.1 & 0.02 & 15.9 \\
20 & 15.7 & 4.7 & 2.8 & 1.8 & 1.0 & 0.9 & 0.8 & 0.7 & 0.2 & 0.03 & 14.6 \\
21 & 11.4 & 3.4 & 2.0 & 1.3 & 0.8 & 0.6 & 0.6 & 0.5 & 0.2 & 0.02 & 13.2 \\
22 & 7.8 & 2.3 & 1.4 & 0.9 & 0.5 & 0.4 & 0.4 & 0.4 & 0.1 & 0.01 & 12.9 \\
23 & 7.3 & 2.2 & 1.3 & 0.9 & 0.5 & 0.4 & 0.4 & 0.3 & 0.1 & 0.01 & 11.3 \\
24 & 11.9 & 3.6 & 2.1 & 1.4 & 0.8 & 0.7 & 0.6 & 0.6 & 0.2 & 0.02 & 22.5 \\
25 & 10.3 & 3.1 & 1.8 & 1.2 & 0.7 & 0.6 & 0.5 & 0.5 & 0.1 & 0.02 & 18.7 \\
& & & & & & & & & & &
\end{tabular}

Note. ${ }^{\text {a }}$ First ten columns, $A(\mathrm{~V})$ to $A(\mathrm{M} 2)$, refer to the extinction values derived using the $A(\mathrm{~V})$ from Schlegel et al. (1998).

Table 3

Extinction-Corrected $^{\text {a }}$ Aperture Flux Densities in MilliJansky of Spitzer Galaxies in the ZoA

\begin{tabular}{|c|c|c|c|c|c|c|c|c|c|}
\hline ID & $\mathrm{J} 1.25$ & H1.65 & Ks2.17 & IRAC3.6 & IRAC4.5 & IRAC5.8 & IRAC8 & MIPS24 & MIPS70 \\
\hline 1 & 6.9 & 6.7 & 2.1 & 3.530 & 2.120 & 3.275 & 7.109 & b & b \\
\hline 2 & 22.5 & 20.7 & 15.7 & 9.315 & 6.236 & 19.397 & 14.117 & 45.9 & \\
\hline 3 & 155.0 & 55.2 & 41.7 & 22.848 & 17.238 & 40.437 & 106.756 & 88.4 & 791.7 \\
\hline 4 & 848.0 & 117.0 & 30.1 & 9.167 & 5.866 & 5.907 & 28.262 & 9.9 & $\ldots$ \\
\hline 5 & 339.0 & 81.7 & 24.9 & 10.866 & 8.078 & 21.896 & 71.899 & 35.7 & $\ldots$ \\
\hline 6 & 401.0 & 87.6 & 29.2 & 10.316 & 7.196 & 9.225 & 25.140 & 11.9 & $\ldots$ \\
\hline 7 & 3290.0 & 349.0 & 91.2 & 16.589 & 10.096 & 2.152 & 15.965 & 8.5 & $\ldots$ \\
\hline 8 & 38.0 & 31.4 & 21.2 & 12.202 & 8.212 & 20.278 & 56.766 & 49.3 & 663.0 \\
\hline 9 & 72.5 & 28.7 & 11.9 & 6.306 & 4.060 & 4.154 & 16.271 & 9.4 & .. \\
\hline 10 & 754.0 & 124.0 & 46.0 & 11.660 & 8.217 & 8.161 & 29.893 & 8.5 & 179.4 \\
\hline 11 & 34.6 & 16.3 & 3.7 & 3.484 & 2.014 & 4.197 & 6.766 & 3.4 & $\ldots$ \\
\hline 12 & 1840.0 & 138.0 & 31.6 & 13.225 & 8.223 & 8.541 & 33.679 & 10.2 & 156.0 \\
\hline 13 & 16.7 & 5.0 & 3.0 & 2.761 & 1.917 & 0.534 & 7.889 &..$^{b}$ &.$^{\mathrm{b}}$ \\
\hline 14 & 545.0 & 105.0 & 28.2 & 14.223 & 9.692 & 14.856 & 37.765 & 25.5 & 280.8 \\
\hline 15 & 14.9 & 2.9 & 4.6 & 2.926 & 1.946 & 2.379 & 9.957 & 5.1 & 62.4 \\
\hline 16 & 59.6 & 33.5 & 35.8 & 9.162 & 6.077 & 5.782 & 28.286 & 15.6 & 89.7 \\
\hline 17 & 25.6 & 12.1 & 7.9 & 3.793 & 2.826 & 3.832 & 17.482 & $\ldots{ }^{b}$ & $\ldots{ }^{b}$ \\
\hline 18 & 26.9 & 16.2 & 11.2 & 3.997 & 2.931 & 1.145 & 11.450 & 3.7 & $\cdots$ \\
\hline 19 & 16.5 & 12.6 & 9.2 & 6.156 & 4.527 & 7.414 & 27.557 & 16.2 & 328.0 \\
\hline 20 & 99.9 & 27.6 & 11.9 & 3.892 & 3.471 & 6.628 & 19.335 & 8.8 & 216.1 \\
\hline 21 & 7.9 & 5.2 & 2.6 & 2.435 & 0.885 & 2.276 & 5.383 & 4.3 & $\ldots$ \\
\hline 22 & 14.1 & 8.8 & 6.0 & 3.976 & 2.383 & 2.308 & 8.040 & 5.5 & $\ldots$ \\
\hline 23 & 8.2 & 6.7 & 6.9 & 3.912 & 2.917 & 3.508 & 10.329 & 5.4 & $\ldots$ \\
\hline 24 & 32.8 & 14.6 & 5.3 & 4.899 & 2.948 & 5.066 & 13.529 & 6.4 & $\ldots$ \\
\hline 25 & 32.6 & 14.4 & 5.1 & 4.700 & 2.700 & 4.800 & 13.333 & 6.3 & $\ldots$ \\
\hline
\end{tabular}

Notes.

${ }^{\text {a }}$ Based on Schlegel et al. (1998).

b Outside of MIPS survey. 

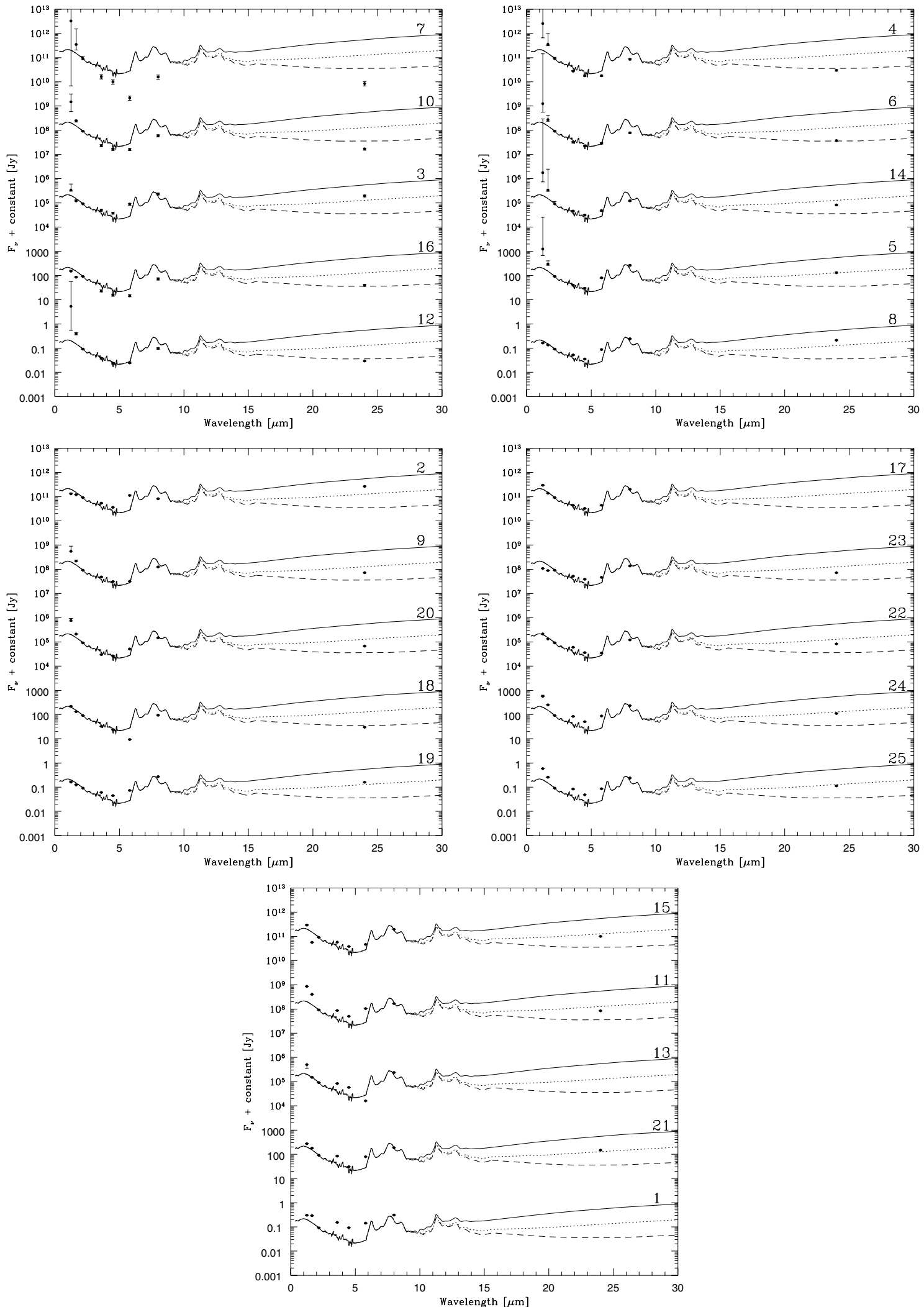

Figure 5. Extinction-corrected SEDs, based on the IRAS/DIRBE maps (Schlegel et al. 1998), of the 25 candidate galaxies (see Table 3). The number associated with each SED is the ID of the galaxy as listed in Table 1. From top to bottom, the SEDs are ordered in decreasing Ks-band magnitude and a constant is added to their measured flux densities to allow for direct comparison. Models of Dale \& Helou (2002) with $\alpha=1.5$ (solid line), 2.0 (dotted line), and 4.0 (dashed line). The models have been normalized to the $K$ s magnitude of each galaxy and shifted in the $y$-axis for easy comparison.

et al. (2005) to be 280.9 (channel 1), 179.7 (channel 2), 115.0 (channel 3), and $64.13 \mathrm{Jy}$ (channel 4). Finally, the extinction corrections at these wavelengths were obtained using the transformation given in Indebetouw et al. (2005) (their Equation (4); 

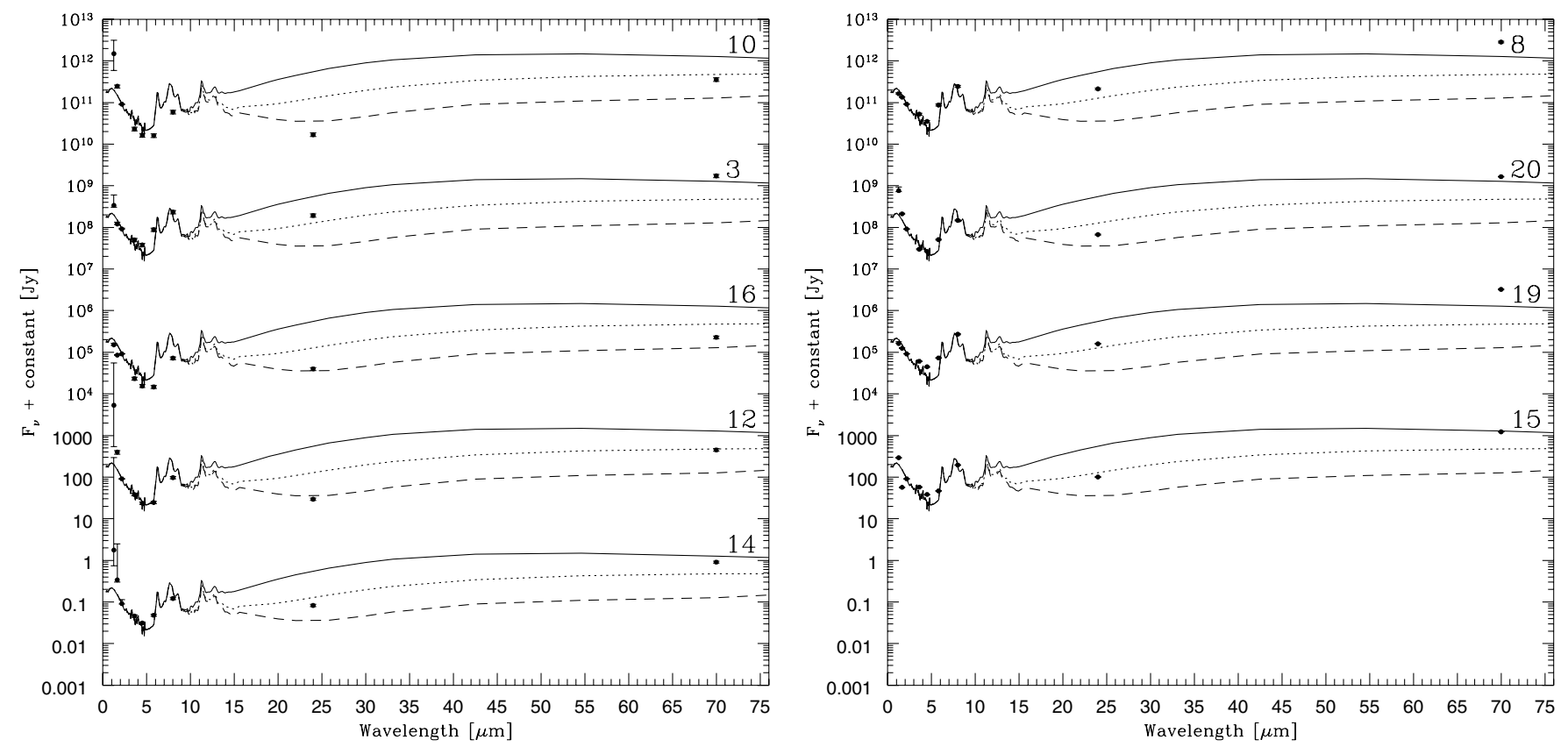

Figure 6. Extinction-corrected SEDs, based on the IRAS/DIRBE maps (Schlegel et al. 1998), of galaxies with measured $70 \mu \mathrm{m}$ flux densities. The models shown are the same as in Figure 5.

see Table 2 for computed values). The aperture correction was applied to the MIPS flux densities following the MIPS data handbook prescription (Engelbracht et al. 2007; Gordon et al. 2007). The aperture correction for a $6^{\prime \prime}$ radius aperture was 1.7 at $24 \mu \mathrm{m}$ and 3.9 at $70 \mu \mathrm{m}$. The extinction corrections at these wavelengths were derived using the extinction curve of Indebetouw et al. (2005) and are listed in Table 2.

All extinction-corrected photometric measurements are given in Table 3. Uncertainties in the flux density measurements varied with passband. In the shorter 2MASS wavelengths, the photometric uncertainties were dominated by the extinction correction. The upper and lower error bars on the 2MASS flux density measurements were computed using the maximum and minimum values of $A(\mathrm{~V})$ from Schlegel et al. (1998) derived using a $5^{\prime}$ radius circle for averaging centered on the reference position. For the Spitzer bands, we assumed flux density uncertainties of the order of $20 \%$.

\section{SPECTRAL ENERGY DISTRIBUTION}

The SEDs of the 25 candidate galaxies, covering the wavelength range of 1.25-24 $\mu \mathrm{m}$, are shown in Figure 5. From top to bottom, the SEDs are ordered in decreasing Ks-band magnitude and a constant is added to their measured flux densities to allow for direct comparison. For nine of the extragalactic sources, a flux density was also measured in the $70 \mu \mathrm{m}$ images, and these extended SEDs are plotted in Figure 6.

We selected the infrared SED distribution models of normal star-forming galaxies of Dale et al. (2001) and Dale \& Helou (2002) to create generic galaxy SED templates and compare them with the observations. Our main reasons for selecting these models over other ones were that they cover a wide wavelength range, from 3 to $1100 \mu \mathrm{m}$, and are described by a single parameter, $f_{v}(60 \mu \mathrm{m}) / f_{v}(100 \mu \mathrm{m})$. The large wavelength coverage, based on IRAS and ISO measurements (Dale \& Helou 2002), is particularly important for this study as the new sources suffer from significant extinction at shorter wavelengths, making the longer wavelengths' flux density measurements more reliable. The local SEDs in these models are combined assuming a power-law distribution of dust mass over heating intensity in a given galaxy, $d M_{d}(U) \propto U^{-\alpha} d U$, where $d M_{d}(U)$ is the dust mass heated by a radiation field at intensity $U$ and the exponent $\alpha$ is a parameter that represents the relative contributions of the different local SEDs. The range of $\alpha$ that describes the suite of normal galaxy SEDs is approximately $1.0<\alpha<2.5$. The models were obtained from Dale's Web site. ${ }^{9}$ The file contained the model SEDs for a range of $\alpha$ of 0.0625-4.0 (step size of 0.0625).

Three model SEDs are shown in Figures 5 and 6 along with the data points. These model SEDs were generated using a value for the parameter $\alpha$ of 1.5, 2.0, and 4.0 and normalized to the $K$ s flux density of each galaxy. They were chosen to approximately mimic the range of behavior of the measured SEDs. The first striking result of this model-to-data comparison is that the SEDs of all our candidate extragalactic sources are unambiguously consistent with the SEDs of normal galaxies. Moreover, we found that the majority of them, i.e. 23 out of 25 , showed an extended disk at $8 \mu \mathrm{m}$ (see Figure 2). This fraction drops to $50 \%$ in the near-infrared (see Figure 3 ) as the extinction becomes larger at shorter wavelengths. The two sources that appeared to be compact at $8 \mu \mathrm{m}$, source IDs 15 and 16 in Table 1, revealed their disk-like morphology in their 2MASS images.

This selection bias was not a surprise as elliptical galaxies could not be easily distinguished from compact Galactic sources in our visual search given the spatial resolution of the IRAC and MIPS images and their similar infrared colors. Assuming the morphological mix of nearby galaxies observed in the field ( $25 \%$ ellipticals; see, e.g., van den Bergh 1998), we estimated that our search may have missed around eight earlytype galaxies. Needless to say, this fraction increases if the galaxies are in a cluster environment. However, given the

\footnotetext{
9 http://faraday.uwyo.edu/ ddale/research/seds/seds.html.
} 


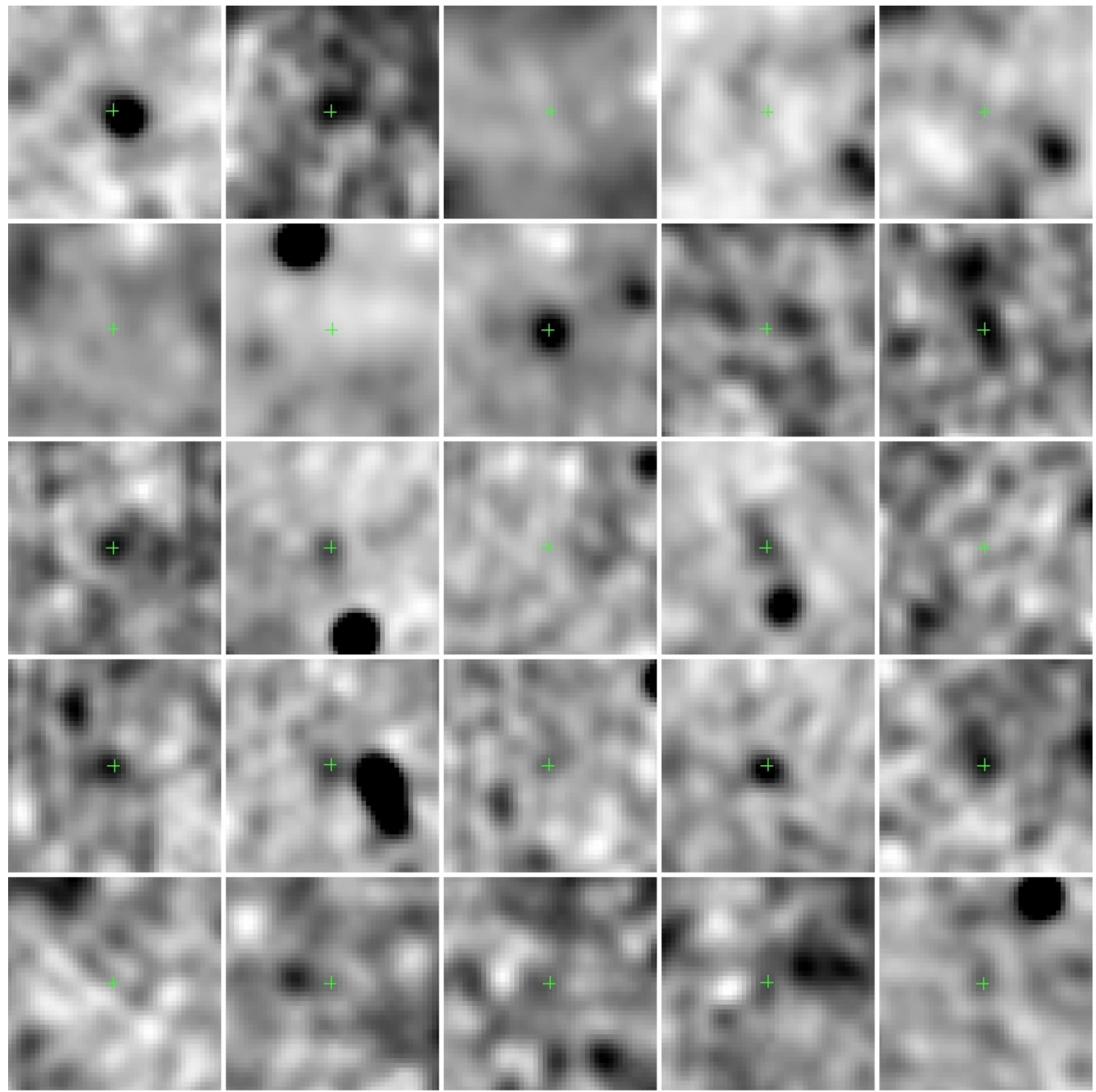

Figure 7. NVSS $1.4 \mathrm{GHz}$ postage stamps of the same galaxies. The galaxies are displayed in the same order as shown in Figure 2 . The FOV of each image is $5.5^{\prime} \times$ $5.5^{\prime}$ (7.5" per pixel) with north up and east to the left. The green crosses overlaid on the images represent the central location of the candidates as listed in Table 1. The images are displayed in reverse color map and the scale parameters of the postage stamps were optimized for each individual source and therefore differ for each image.

growing evidence that as much as $\sim 70 \%$ of the nearby elliptical galaxies contain a significant amount of gas and dust (see, e.g., Combes et al. 2007; Morganti et al. 2006; Jura 1986) in both the field and cluster environments, our mid- to far-infrared search probably only missed around six $(\sim 18 \%)$ dusty early types.

\section{IDENTIFY MEMBERSHIP TO LOCAL LARGE-SCALE STRUCTURE}

\subsection{Location in the 2D Sky}

In order to ascertain the location of the newly discovered galaxies within the two-dimensional local large-scale structure, we first queried the NASA Extragalactic Database (NED) to cross-check these new sources within the existing database and to also identify any previously detected galaxies listed for our survey region $\left(l=40-65^{\circ},|b| \lesssim 1^{\circ}\right.$, and $\left.z<0.08\right)$. The outcome of this search was negative, and no matches were found. However, five additional sources, with no match to ours, were returned by NED and identified as detections from the HI Parkes Zone of Avoidance Survey (HIZOA; Donley et al. 2005) and the HI Parkes All-Sky Survey (HIPASS; Staveley-Smith et al. 2000). Taking into account the $3 \sigma$ positional uncertainty of the HI Parkes surveys of $3^{\prime}$, we searched for the near- to far-infrared counterparts to these sources and found no extended sources at 


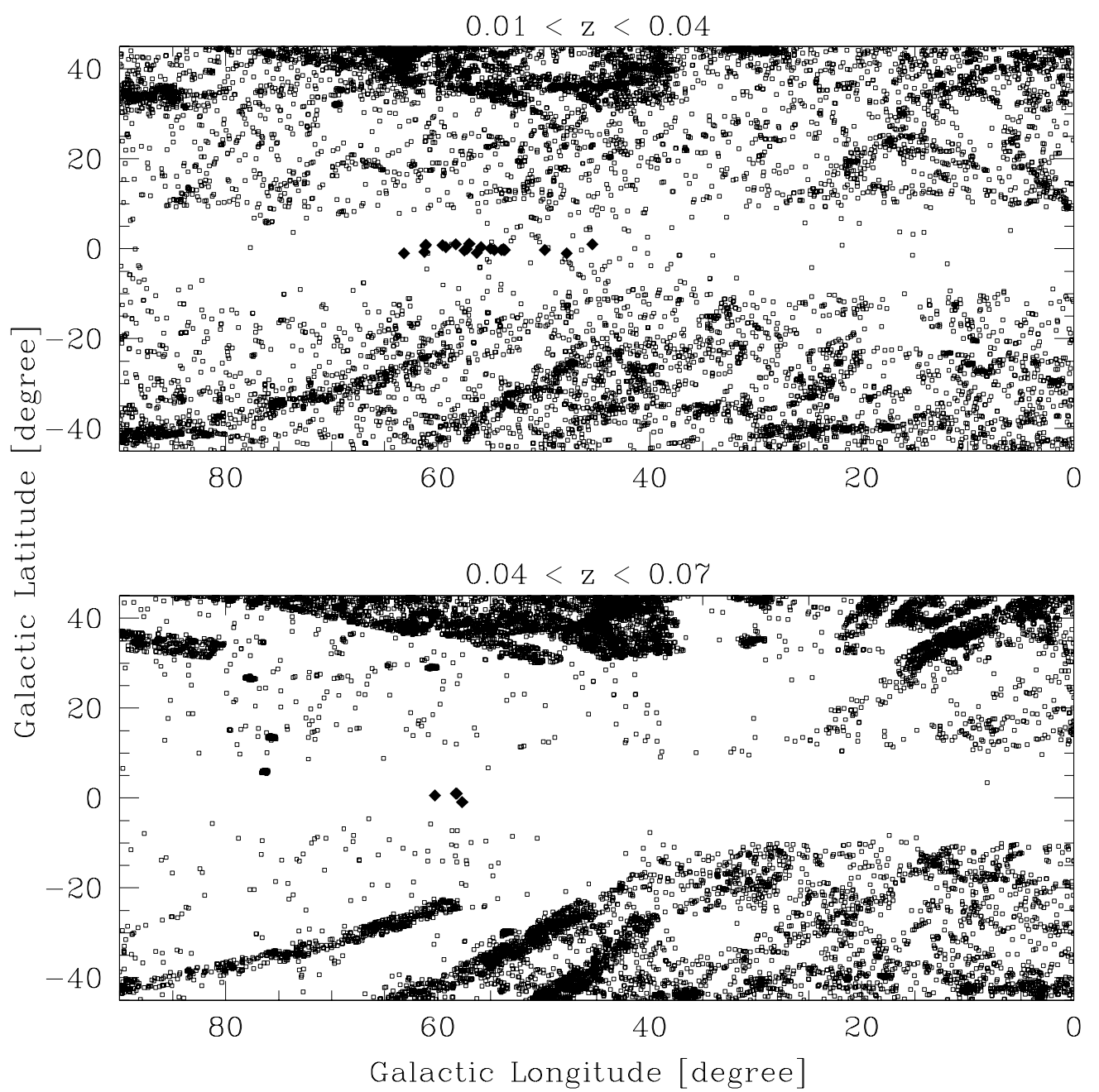

Figure 8. Spatial distribution of the new galaxies (filled lozenges near the Galactic equator) in the $40^{\circ} \geqslant l \geqslant 65^{\circ}$ region superimposed on the distribution of NED galaxies (open squares) in the redshift range $0.01<z<0.04$ (top panel) and $0.04<z<0.07$ (bottom panel). The figure, more specifically the top panel, suggests that the new galaxies are consistent with being spatially located on an extension of the already known large-scale structure at higher Galactic latitude.

these locations. As this first query returned only sources with redshift, we queried NED again at each of the candidate galaxy locations without any redshift constraint and this time we found seven matches (see Table 1), four classified as detections from the NRAO/VLA Sky Survey (NVSS), two from the Westerbork Synthesis Radio Telescope Galactic Plane Survey (WSRTGP), and one from the First White+Giommi+Angelini ROSAT $\mathrm{X}$-Ray sources list (1WGA). The NVSS postage stamps of all the candidates are shown in Figure 7. We carefully examined all NVSS postage stamps and confirmed the four NVSS and two WSRTGP detections returned by NED as well as an additional five detections, bringing the total to 11 sources with believable detections at $1.4 \mathrm{GHz}$. The 1WGA source returned from NED did not have a detection at $1.4 \mathrm{GHz}$.

We then looked at the location of the discovered galaxies within the two-dimensional distribution of the known galaxies in the local universe, i.e. we extended our search to a larger area in the sky $\left(l=0^{\circ}-90^{\circ},|b| \leqslant 45^{\circ}\right)$ and within a redshift space of $z=0.01-0.04$ (11,243 galaxies) and $z=0.04-0.07$ (13,055 galaxies). These included the HIZOA galaxies found by Donley et al. (2005). The positions of the new galaxies are overlaid on the map of the local large-scale structure in Figure 8, top and bottom panels, respectively. We find that these overdensities of galaxies are located in the sky near a local large- scale structure, seen also in the 2MASS extended source catalog (Jarrett 2004) for redshifts $z=0.01-0.03$, and extending from $|b|=4^{\circ}-40^{\circ}$. Our findings therefore provide the first strong evidence of a bridging at very low Galactic latitude between the two large-scale structures on both sides of the Galactic plane.

\subsection{Distance/Redshift Determination}

Although the overdensities of galaxies which we have discovered appear to be near a filament, this does not necessarily prove their membership. However, a photometric redshift estimate can certainly narrow down the probability that these discovered galaxies are part of this local superstructure.

We were able to estimate the distance and therefore redshift to our candidate galaxies using two independent methods. The first method simply made use of the Ks-band magnitudes of the sources and assumed the SED of an $\mathrm{L}^{*}$ galaxy, with $M_{K}^{*}=-24$ mag obtained from the $K$-band field luminosity function (Kochanek et al. 2001). The SEDs normalized to the $K$ s-band are shown in Figure 5. The inferred distances are 54$356 \mathrm{Mpc}$. This puts our galaxies in the $4050-26700 \mathrm{~km} \mathrm{~s}^{-1}$ velocity range, or at a redshift of $0.013-0.084$ (in our calculations, we assumed a cosmology with $H_{0}=75 \mathrm{~km} \mathrm{~s}^{-1} \mathrm{Mpc}^{-1}$, $\Omega_{M}=0.3$, and $\Omega_{\Lambda}=0.7$ ). 

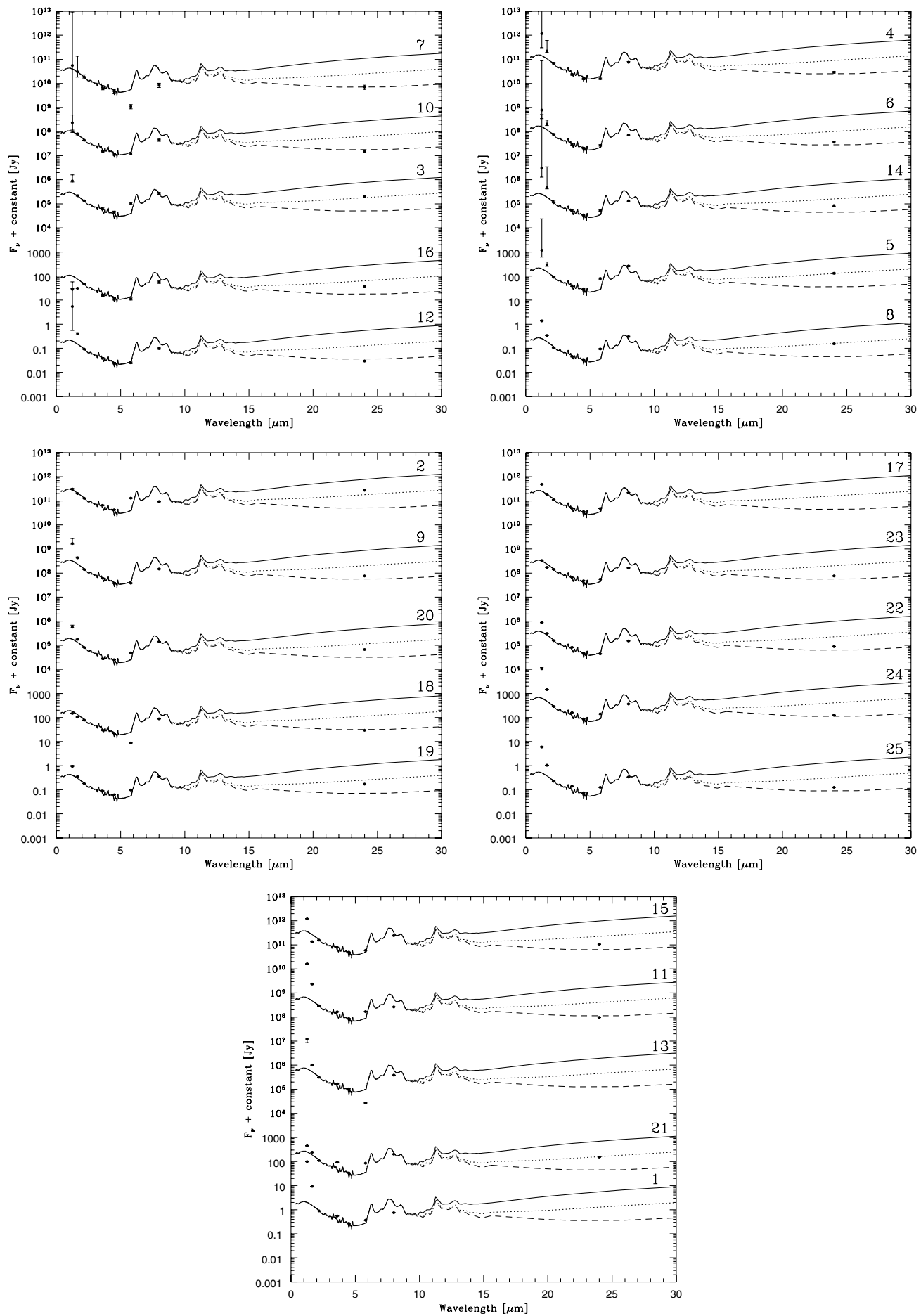

Figure 9. Extinction-corrected SEDs, based on our SED fit, of the 25 candidate galaxies (see Table 4). The models shown are the same as in Figure 5. The models have been normalized to the IRAC 3.6 and $4.5 \mu \mathrm{m}$ bands.

The second and more robust method made use of the full SED, weighted most heavily on the IRAC channel 1 and 2 flux density measurements, which suffered the least from uncertainties associated with extinction (more important at shorter wavelengths) and polycyclic aromatic hydrocarbons (PAHs) emission features (more important in IRAC channels 3 and 4), to estimate the distance to the galaxies. The new fits, weighted to the IRAC channels 1 and 2, are shown in Figure 9, along with the extinction-corrected flux densities obtained using the SED fit (see Table 4). The redshifts and new values for the visual extinction derived using this more robust method are listed in Tables 5 and 2 (last column), respectively. The galaxies found in this region of the sky have estimated redshifts ranging between 0.016 and 0.068 (distance of 66$288 \mathrm{Mpc}$ ).

As seen in Table 2 (first and last columns) the $A(\mathrm{~V})$ values derived from the SED fits are sometimes significantly different than the IRAS/DIRBE extinction values. For eight of the 25 galaxy candidates (source IDs 4, 5, 6, 7, 10, 16, 18, and 20), the $A(\mathrm{~V})$ values derived from the SED fits are lower than those given by Schlegel et al. (1998) by a median value of $2.8 \mathrm{mag}$, with the largest difference being the value of $14.7 \mathrm{mag}$ 
Table 4

Extinction-Corrected $^{\text {a }}$ Aperture Flux Densities in MilliJansky of Spitzer Galaxies in the ZoA

\begin{tabular}{rrrrrrrrrr}
\hline \hline ID & \multicolumn{1}{c}{ J1.25 } & H1.65 & Ks2.17 & \multicolumn{1}{c}{ IRAC3.6 } & IRAC4.5 & IRAC5.8 & IRAC8 & MIPS24 & MIPS70 \\
\hline 1 & 2265.7 & 214.2 & 20.4 & 12.576 & 6.230 & 8.411 & 17.233 & $\ldots$ b & $\ldots$ \\
2 & 53.1 & 34.6 & 22.0 & 11.247 & 7.317 & 22.310 & 16.098 & 47.7 & $\ldots$ \\
3 & 409.0 & 98.6 & 61.0 & 28.265 & 20.648 & 47.356 & 123.820 & 92.3 & 796.3 \\
4 & 390.2 & 73.6 & 22.2 & 7.732 & 5.077 & 5.206 & 25.101 & 9.6 & $\ldots$ \\
5 & 320.7 & 79.0 & 24.4 & 10.735 & 7.995 & 21.699 & 71.292 & 35.6 & $\ldots$ \\
6 & 250.3 & 66.1 & 24.3 & 9.303 & 6.592 & 8.544 & 23.393 & 11.7 & $\ldots$ \\
7 & 55.9 & 30.5 & 18.5 & 6.788 & 4.730 & 1.108 & 8.564 & 7.1 & $\ldots$ \\
8 & 68.0 & 44.5 & 26.6 & 13.863 & 9.151 & 22.294 & 62.048 & 50.6 & 665.3 \\
9 & 219.8 & 55.7 & 18.4 & 8.042 & 4.990 & 4.976 & 19.276 & 9.9 & $\ldots$ \\
10 & 117.7 & 40.8 & 22.2 & 7.759 & 5.816 & 6.031 & 22.506 & 7.8 & 177.4 \\
11 & 653.6 & 94.5 & 11.7 & 6.636 & 3.479 & 6.772 & 10.602 & 3.9 & $\ldots$ \\
12 & 1891.7 & 140.3 & 31.9 & 13.306 & 8.266 & 8.580 & 33.822 & 10.2 & 156.0 \\
13 & 404.9 & 33.6 & 10.5 & 5.555 & 3.469 & 0.897 & 12.842 & $\ldots$ & $\ldots$ \\
14 & 948.8 & 146.3 & 35.0 & 16.062 & 10.745 & 16.259 & 41.105 & 26.1 & 281.7 \\
15 & 61.3 & 6.8 & 8.0 & 3.989 & 2.532 & 2.995 & 12.359 & 5.4 & 62.9 \\
16 & 11.0 & 12.2 & 18.4 & 6.323 & 4.437 & 4.391 & 21.844 & 14.5 & 88.8 \\
17 & 42.2 & 16.3 & 9.6 & 4.232 & 3.101 & 4.156 & 18.867 & $\ldots$ & $\ldots$ \\
18 & 18.2 & 12.8 & 9.6 & 3.671 & 2.727 & 1.075 & 10.791 & 3.6 & $\ldots$ \\
19 & 94.6 & 35.8 & 18.2 & 9.029 & 6.265 & 9.852 & 35.988 & 17.5 & 331.4 \\
20 & 73.6 & 23.0 & 10.6 & 3.640 & 3.280 & 6.307 & 18.455 & 8.7 & 215.7 \\
21 & 13.0 & 7.0 & 3.2 & 2.717 & 0.971 & 2.469 & 5.810 & 4.4 & $\ldots$ \\
22 & 58.0 & 20.5 & 10.4 & 5.421 & 3.100 & 2.905 & 9.979 & 5.9 & $\ldots$ \\
23 & 24.9 & 13.0 & 10.7 & 4.989 & 3.585 & 4.202 & 12.237 & 5.7 & $\ldots$ \\
24 & 619.6 & 84.6 & 16.8 & 9.331 & 5.093 & 8.174 & 21.199 & 7.3 & $\ldots$ \\
25 & 334.6 & 58.0 & 12.7 & 7.832 & 4.164 & 7.013 & 19.032 & 7.0 & $\ldots$ \\
\hline
\end{tabular}

Notes.

${ }^{\text {a }}$ Based on our SED fits.

${ }^{b}$ Outside of MIPS survey.

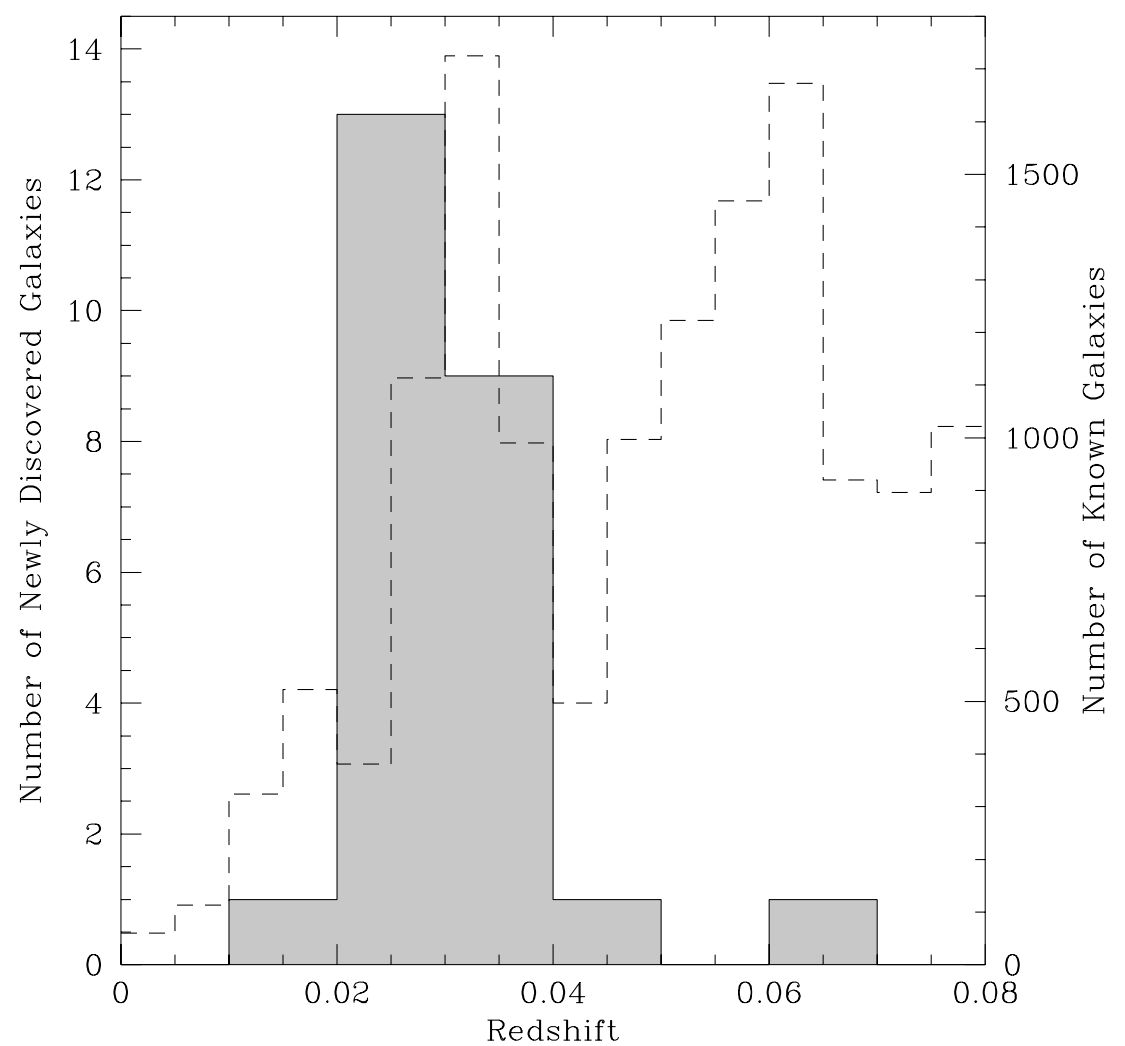

Figure 10. Left axis: redshift histogram (gray shaded and solid line) of our 25 newly discovered galaxies. Right axis: redshift histogram (dashed line) of all known galaxies from NED in the region of the Galactic plane with $40^{\circ}<l<70^{\circ}$ and $-45^{\circ}<b<45^{\circ}$ with $0<z<0.08$. The figure suggests that the new galaxies are consistent in redshift space with an extension of the already known large-scale structure at higher Galactic latitude. 
Table 5

Redshift Estimates of Spitzer Galaxies in the ZoA

\begin{tabular}{lc}
\hline Name & Redshift (SED fit) \\
\hline SPITZER191050+113409 & 0.028 \\
SPITZER192252+124513 & 0.026 \\
SPITZER192404+145632 & 0.016 \\
SPITZER193206+183140 & 0.026 \\
SPITZER193145+181940 & 0.025 \\
SPITZER193133+181516 & 0.025 \\
SPITZER193105+182614 & 0.029 \\
SPITZER193337+190727 & 0.024 \\
SPITZER193320+193334 & 0.029 \\
SPITZER193349+202958 & 0.027 \\
SPITZER193915+201426 & 0.037 \\
SPITZER193825+212701 & 0.022 \\
SPITZER193324+214918 & 0.038 \\
SPITZER193956+213017 & 0.021 \\
SPITZER194223+212514 & 0.044 \\
SPITZER193633+225125 & 0.029 \\
SPITZER193608+225054 & 0.040 \\
SPITZER193632+225355 & 0.040 \\
SPITZER193958+235251 & 0.029 \\
SPITZER194042+232429 & 0.038 \\
SPITZER194211+242507 & 0.068 \\
SPITZER194257+251849 & 0.038 \\
SPITZER194349+251449 & 0.038 \\
SPITZER194919+243738 & 0.031 \\
SPITZER195443+260745 & 0.035 \\
\hline &
\end{tabular}

(source ID 7). A similar IRAS/DIRBE overestimate of the Galactic visual extinction values was reported by Nagayama et al. (2004) and Schröder et al. (2007) in the direction of the highly obscured radio-bright galaxy PKS 1343-601 at Galactic coordinates of $(l, b)=\left(309.7^{\circ}, 1.7^{\circ}\right)$. However, our comparison indicates that for the remaining 17 galaxies the $A(\mathrm{~V})$ value derived is larger than the IRAS/DIRBE value, by a median value of 4.0 mag and with the largest difference being the value of $20.8 \mathrm{mag}$ (source ID 1). This suggests that the IRAS/DIRBE maps may be underestimating the amount of extinction for the most obscured regions $\left(|b| \leqslant 1^{\circ}\right)$ of the Milky Way.

As can be seen by looking at the postage stamps, our candidate galaxies are all fainter (after the flux densities have been corrected for dust extinction) than the two galaxies discussed in Jarrett et al. (2007). Indeed, the IRAC channel 1 flux densities of our sample range from 2.4 to $22.8 \mathrm{mJy}$ (see Table 3 ) whereas the two galaxies discussed in Jarrett et al. (2007) have flux densities of 35.0 and $18.3 \mathrm{mJy}$ (see their Table 1). Therefore, our galaxies probe flux densities ten times fainter than those of Jarrett et al. (2007). It follows that we are probing deeper in redshift space, $z=0.016-0.068$ (see Table 5), beyond the $z \simeq 0.015$ galaxies of Jarrett et al. (2007). It is therefore not surprising that for the majority of the candidate galaxies, we did not find an HI counterpart. By comparing the properties of our galaxy with the largest IRAC channel 1 and 2 flux densities, source ID 3 (22.8 and $17.2 \mathrm{mJy}$, see Table 3), with Jarrett et al.'s two galaxies (G1: 35.0 and $24.1 \mathrm{mJy}, \mathrm{G} 2: 18.3$ and $12.7 \mathrm{mJy}$, see their Table 1 ), we find that our photometric redshift estimate of $z=0.016$ for this galaxy is entirely consistent with the $\mathrm{HI}$ radial velocity redshifts derived for Jarrett et al.'s galaxies $(z \simeq 0.015)$.

A histogram of the redshifts of the 25 discovered galaxies is shown in Figure 10. We find that the majority of them occupy the redshift range $z \simeq 0.01-0.05$, with one source located at $z \simeq 0.07$. For comparison, the redshift distribution of all known galaxies in the redshift range $z=0.0-0.08$ and with $l=40-70^{\circ}$ and $|b| \leqslant 45^{\circ}$, i.e. 13907 galaxies, is also shown

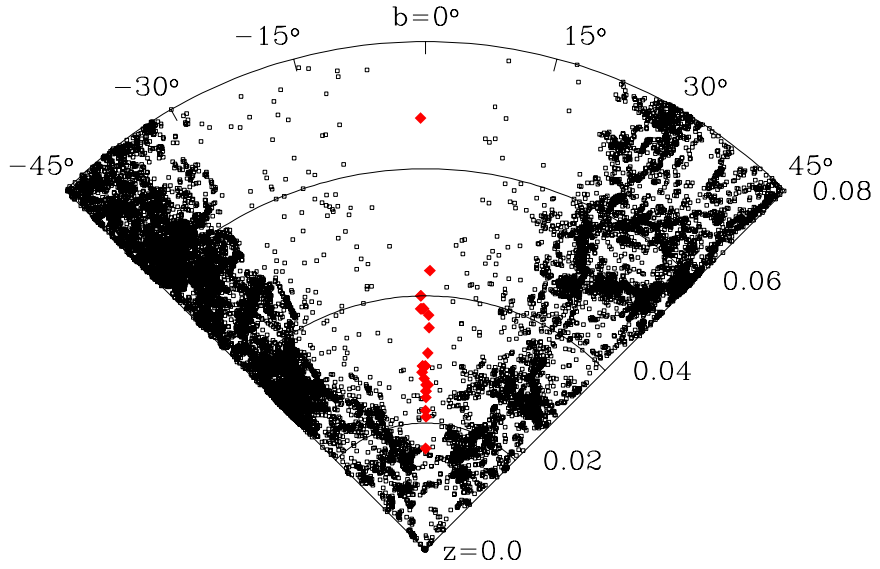

$40^{\circ}<1<70^{\circ}$

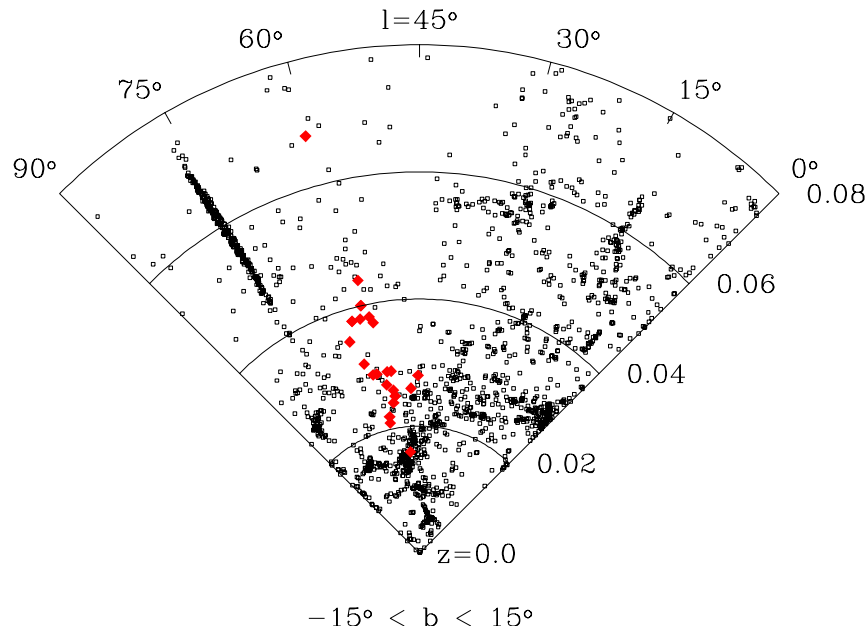

Figure 11. Cone plot in redshift space of the NED galaxies (open squares) located in a slice of $-45^{\circ}<b<45^{\circ}$ for Galactic longitudes in the range $40^{\circ}<l<70^{\circ}$ (top panel), including the new galaxies of this study (filled red lozenges). The bottom panel shows the cone diagram with $0^{\circ}<l<90^{\circ}$ for Galactic latitudes in the range $-15^{\circ}<b<15^{\circ}$. The overall distribution of these new objects is consistent with the local superstructures found between the redshifts of $z=0-0.08$.

in Figure 10. The overdensity in redshift space of our sample agrees remarkably well with the one of the previously known local large-scale structure in this region (see Figure 11). This seems to agree again with the interpretation that these newly revealed galaxies belong to an extension to lower Galactic latitude of the already known local superstructure and belong to a bridge extending the filamentary structure seen on each side of the Galactic plane. However, remember that our redshift determination depends on the assumption that these are all $\mathrm{L}^{*}$ galaxies. If they are not, then clearly their redshift distribution will change. For example, it is possible that the galaxy with the largest redshift is not an $\mathrm{L}^{*}$ galaxy but somewhat fainter and that we are overestimating its distance.

\section{SUMMARY}

We reported in this paper the discovery of 25 galaxies in the ZoA located around $l \sim 47^{\circ}$ and $55^{\circ}$ and $|b| \lesssim 1^{\circ}$ in the Sagitta-Aquila region. These overdensities are consistent with the local large-scale structure found at similar Galactic longitude and extending from $|b| \sim 4^{\circ}$ to $40^{\circ}$. We presented their SEDs and provided evidence of their extragalactic nature. 
Their redshifts were estimated using the infrared photometric measurements obtained from 2MASS and Spitzer. We found that the majority of the newly discovered galaxies occupy the redshift range $z \simeq 0.01-0.05$, with one source located at $z \simeq 0.07$. A comparison with known sources in the local universe revealed that these galaxies are located at similar overdensities in redshift space. This seems to imply that these newly discovered galaxies belong to an extension to lower Galactic latitude of the already known local superstructure, providing a first view of the bridging between both sides of the Galactic plane.

However, we must emphasize that these photometric redshifts, which suffer from the uncertainties associated with the extinction correction and are based on the assumption that these are all $\mathrm{L}^{*}$ galaxies, can only be determined with certainty using spectroscopic data. Therefore, we are carrying out follow-up near-infrared spectroscopic observations of the newly discovered galaxies with ground-based telescopes. The spectroscopic redshifts will allow us to derive a better estimate of the amount of extinction in the direction of our sources, which is highly uncertain. In addition, with their distance fixed, we will be able to calculate their intrinsic luminosities and estimate their stellar masses.

In conclusion, the work presented here clearly demonstrates the power of mid- to far-infrared surveys in finding galaxies in highly obscured regions such as the plane of our Galaxy, surveying even the lowest Galactic latitudes of $|b| \lesssim 1^{\circ}$. Moreover, the relative success of our survey implies that the ZoA should no longer be avoided and further studies should be undertaken to expand our knowledge of the local large-scale structure in this heavily obscured part of the universe.

We thank an anonymous referee for helpful suggestions on improving the manuscript. This work is based in part on observations made with the Spitzer Space Telescope, which is operated by the Jet Propulsion Laboratory, California Institute of Technology, under NASA contract 1407.

\section{REFERENCES}

Benjamin, R. A., et al. 2003, PASP, 115, 953

Burgdorf, M. J., et al. 2005, AdSpR, 36, 1050
Colless, M. 1999, RSPTA, 357, 105

Combes, F., Young, L. M., \& Bureau, M. 2007, MNRAS, 377, 1795

Dale, D. A., \& Helou, G. 2002, ApJ, 576, 159

Dale, D. A., Helou, G., Contursi, A., Silbermann, N. A., \& Kolhatkar, S. 2001, ApJ, 549, 215

Donley, J. L., et al. 2005, AJ, 129, 220

Dressler, A., Faber, S. M., Burstein, D., Davies, R. L., Lynden-Bell, D., Terlevich, R. J., \& Wegner, G. 1987, ApJ, 313, 37

Dutra, C. M., Santiago, B. X., \& Bica, E. 2002, A\&A, 381, 219

Engelbracht, C. W., et al. 2007, PASP, 119, 994

Fazio, G. G., et al. 2004, ApJ, 154, 10

Geller, M. J., \& Huchra, J. P. 1989, Science, 246, 897

Gordon, K., et al. 2007, PASP, 119, 1019

Henning, P. A., et al. 2000, AJ, 119, 2686

Indebetouw, R., et al. 2005, ApJ, 619, 931

Jarrett, T. H. 2004, PASA, 21, 396

Jarrett, T. H., Chester, T., Cutri, R., Schneider, S., Rosenberg, J., Huchra, J. P., \& Mader, J. 2000, AJ, 120, 298

Jarrett, T. H., et al. 2007, AJ, 133, 979

Jura, M. 1986, ApJ, 306, 483

Kochanek, C. S., et al. 2001, ApJ, 560, 566

Kolatt, T., Dekel, A., \& Lahav, O. 1995, MNRAS, 275, 797

Kraan-Korteweg, R. 2005, RevMexAA, 18, 48

Kraan-Korteweg, R., \& Lahav, O. 2000, A\&ARv, 10, 211

Meade, M. R., et al. 2007a, "GLIMPSEI-v2.0 Data Release"

Meade, M. R., et al. 2007b, "GLIMPSEII-v1.0 Data Products and Delivery"

Morganti, R., et al. 2006, MNRAS, 371, 157

Nagayama, T., et al. 2004, MNRAS, 354, 980

Radburn-Smith, D. J., Lucey, J. R., Woudt, P. A., Kraan-Korteweg, R. C., \& Watson, F. G. 2006, MNRAS, 369, 1131

Reach, W. T., et al. 2005, PASP, 117, 978

Rieke, G. H., et al. 2004, ApJ, 154, 25

Roman, A. T., Iwata, I., \& Saitō, M. 2000, ApJS, 127, 27

Schlegel, D. J., Finkbeiner, D. P., \& Davis, M. 1998, ApJ, 500, 525

Schröder, A. C., Kraan-Korteweg, R. C., \& Mamon, G. A. 1999, PASA, 16,42

Schröder, A. C., Mamon, G. A., Kraan-Korteweg, R. C., \& Woudt, P. A. 2007, A\&A, 466, 481

Shectman, S. A., Landy, S. D., Oemler, A., Tucker, D. L., Lin, H., Kirshner, R. P., \& Schechter, P. L. 1996, ApJ, 470, 172

Skrutskie, M. F., et al. 2006, AJ, 131, 1163

Staveley-Smith, L., Koribalski, B. S., Stewart, I., Putman, M. E., Kilborn, V. A., \& Webster, R. L. 2000, in ASP Conf. Ser. 217, "Imaging at Radio through Submillimeter Wavelengths", ed. J. G. Mangum, \& S. J. E. Radford (San Francisco, CA: ASP)

Takata, T., Yamada, T., \& Saito, M. 1996, ApJ, 457, 693

van den Bergh, S. 1998, Galaxy Morphology and Classification (Cambridge: Cambridge Univ. Press)

Werner, M. W., et al. 2004, ApJS, 154, 1 